\title{
Access and authentication for multitasking system using 1-wire technology
}

\author{
Apurva Pote ${ }^{1}$, Dinesh Rotake ${ }^{2}$, Mamta Mahajan ${ }^{3}$ \\ ${ }^{1}$ (Electronics and Telecommunication, Nagpur University, India) \\ ${ }^{2}$ (Electronics and Telecommunication, Nagpur University, India) \\ ${ }^{3}$ (Electronics and Telecommunication, Nagpur University, India)
}

\begin{abstract}
This paper presents the design of multiaccess system using ARM and iButton. The system performs applications like identification and verification using aadhaar card, toll access control and electronic money transaction, displays vehicle registration details and also gives access to the vehicle. This system becomes very secure and reliable solution to many problems and has hardware simplicity which is specific to the 1-wire technology. The key part of the whole system is iButton. The iButton is a computer chip enclosed in a 16mm thick stainless steel can. Because of this unique and durable container, up-to-date information can travel with a person or object anywhere they go. Various components used in this system are 1-wire network, keypad, iButton, motor, relay, EEPROM memories.
\end{abstract}

Keywords: ARM processor, iButton, iButton reader, LCD display, 1 -wire communication protocol.

\section{Introduction}

We need to carry different cards for the identification and for accessing different services. Such type of cards provides access to e-commerce facilities, issuing of books in library, authentication to access authorized areas using aadhaar card. For each purpose we have implemented a specific card like for driving a vehicle we need driving license that is based on chip card technology, for college or office purpose we need an identity card based on either RFID technology or bar codes and for e-money we need debit or credit cards based on magnetic stripes. Rather than this we need to carry most frustrating, loose changes for purchasing goods and services.

But now, the iButton is set to change all this issues. Instead of using cash for any services or for authentication purpose, all we need is an iButton. The steel iButton can be mounted virtually anywhere because it is rugged enough to withstand critical situations, outdoors or indoors. It is portable to attach to a ring, watch or key fob and thus facilitates transfer of e-cash from one iButton to another. For this purpose all we need is an inexpensive iButton reader attached to the parallel or serial port of hardware.

In comparison with smart cards, the iButton is indestructible. An iButton has an exceptional life expectancy. And hence the multitasking system containing applications like identification and authorization using aadhaar card, Toll access control and vehicle registration details and access offers superior privacy over conventional technologies.

\section{1 iButton}

\section{Materials and methods}

The keypart of the whole system is "iButton" which is a proprietary technology from maxim. The iButton is a computer chip enclosed in a $16 \mathrm{~mm}$ thick stainless steel can. Because of this unique and durable container, up to date information can travel with a person anywhere they go. An iButton's 64-bit address provides a simple, secure way of identifying a location or an item. It can serve as an electronic serial number that is never duplicated. The durable iButton can be reprogrammed and reused for many years significantly reducing operating costs.

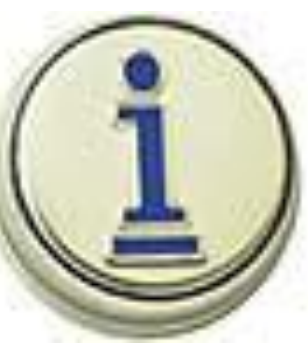

\subsection{1-wire network}

IButton mainly includes base for ground contact and lid for 1-wire data contact. Silicon chip is connected with these contacts. Parasitic power known as data line provides the energy required for the operation to perform. Two metal probe contacts are connected to the circuit which can be used for reading information within the iButton. IButton has very large memory capacity. IButton consists of a unique electronic serial number and identification number and hence iButton is a system which is unable to copy and secure.

The ROM structure is shown in Fig. It consists of 6-byte serial number and 1-byte family code. 


\begin{tabular}{|l|l|l|}
\hline \multicolumn{2}{|c}{ MSB } & MSB LSB \\
\begin{tabular}{|l|l|l|}
\hline B BIT \\
CRC \\
Code
\end{tabular} & 48 BIT Serial Number & $\begin{array}{l}\text { Family } \\
\text { Code }\end{array}$ \\
\hline MSB LSB & LSB & MSB LSB \\
\hline
\end{tabular}

Fig.1 ROM structure

\subsection{LPC 2148 microcontroller}

ARM Processor based LPC 2148 microcontroller is used in this project.

1) The most important feature of ARM is its operating voltage is $3-3.6 \mathrm{~V}$.

2) LPC 2148 has two types of memories such as $8 \mathrm{kB}$ to $40 \mathrm{kB}$ of on chip static RAM and $32 \mathrm{kB}$ to $512 \mathrm{kB}$ of onchip flash memory.

3) Reduced instruction set computer processor offers high performance and very low power consumption.

4) ADC's of LPC 2148 provides 2 analogy signals ADC0 and ADC1. ADC1 has 8 channels whereas ADC0 has 6 channels.

5) 12 Mbps data exchange is done by USB device controller which is built in LPC 2148.

\section{Block diagram of the system}

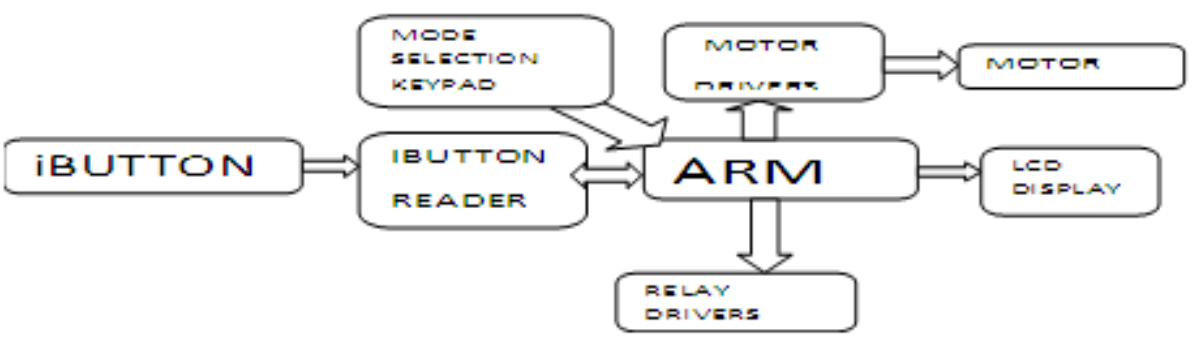

Fig.2 Block diagram of the system

Hardware structure of the system is shown in the above figure. Heart of the system in the above diagram is ARM processor. The system consists of iButton, iButton reader, motor, motor drivers, relay drivers, mode selection keypad, LCD display.

LPC 2148 microcontroller is used which is of 32bit. IButton reader consists of two metal probe contacts connected to the electronic reader. After accessing the iButton it can be used for verification purpose.

\subsection{Identification and authorization using aadhaar card}

\section{Operation of the system}

In this application we have to touch the iButton to the iButton reader and if the account is matched then only it will display information and gives access. If the account is not matched, then it will display "Select module to operate."

\subsection{Toll access control and electronic money transaction}

Initially the highway is closed with the barrier controlled by the microcontroller. As soon as the vehicle comes, the vehicle owner touches his personal iButton to the iButton reader placed beside the highway. Within a second a system read the id and the balance information of the owner through the iButton by the help of 1-wire protocol executed by the microcontroller.

The data is further given to the microcontroller where an embedded software check the balance and debit the highway fees from his available balance then open the door by driving the motor. There are limit sensors used to take feedback to fully open and closed status of the barrier drive by the motor to ensure safe operation.

\subsection{Vehicle registration details and access}

In this case when we touch iButton to iButton reader, it will match the account. If account is matched then it will display the registration details and gives access to the vehicle. If the match is not found then it will display "Select module to operate." 


\subsection{Application 1}

\section{Flowcharts of the applications}

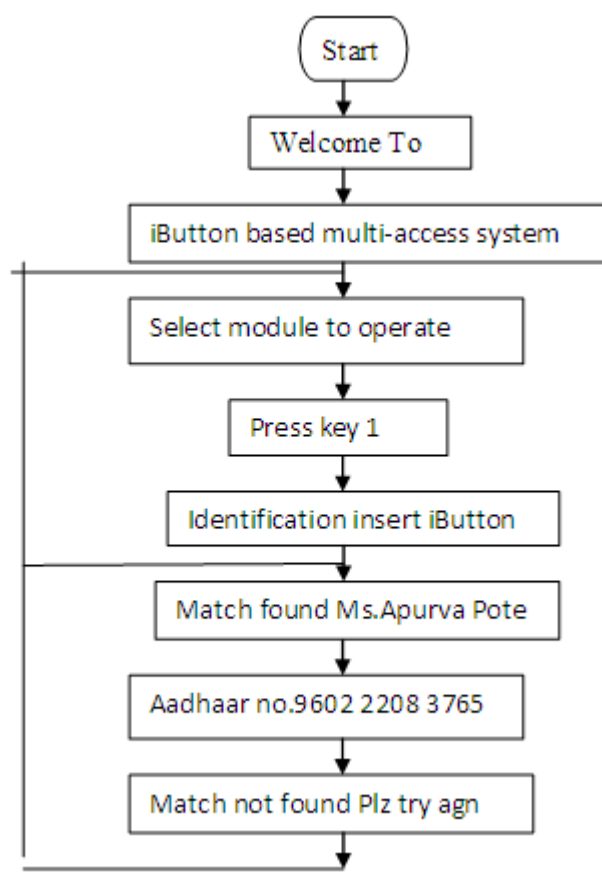

5.2 Application 2

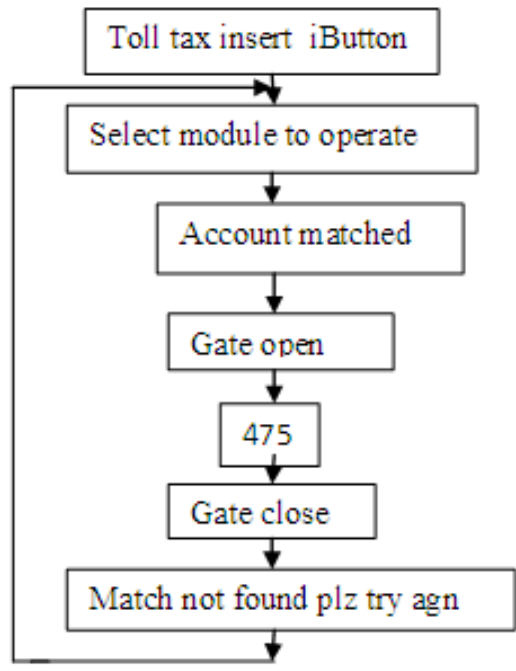

5.3 Application 3

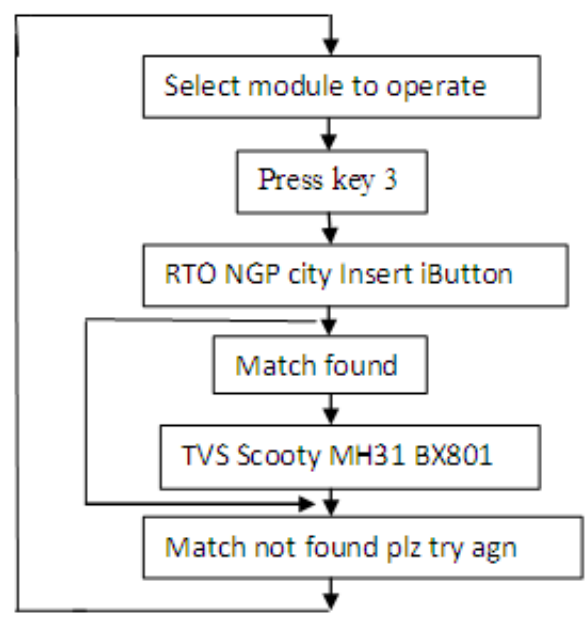




\subsection{Application}

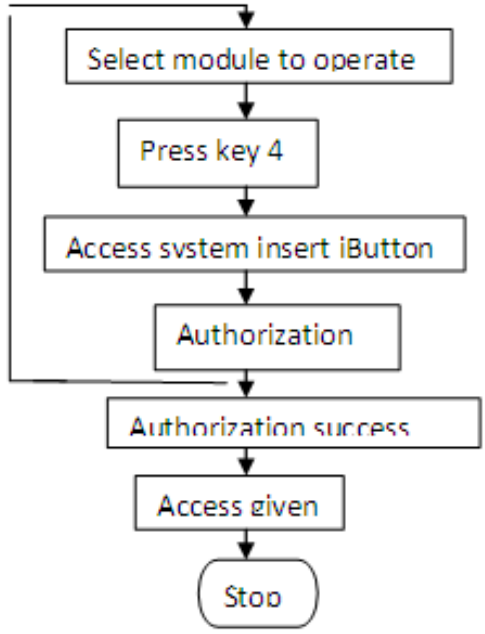

\section{Results}

Fig. 6.1 shows structure of multitasking system.

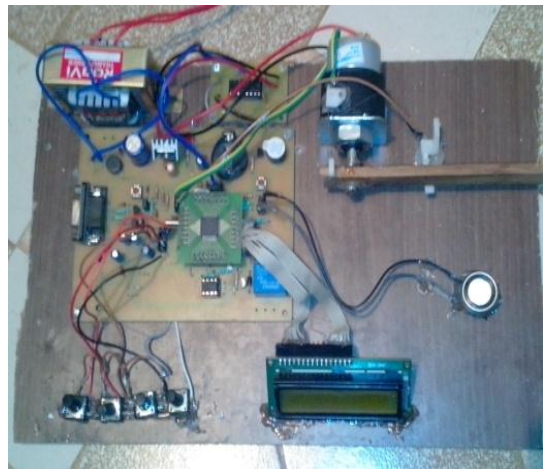

Fig.6.1

Fig.6.2 shows command to select the application. In this result it is shown that, for selecting every application, LCD will display this command. And then we have to press key from keypad.

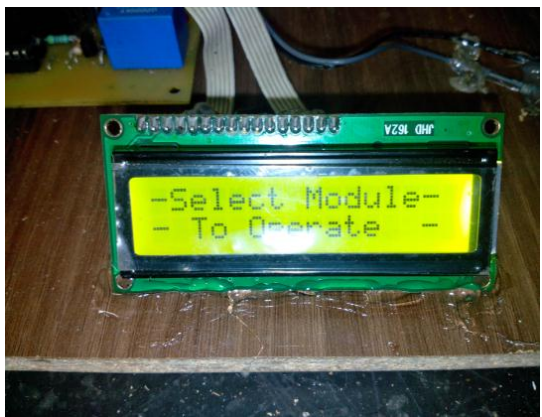

Fig.6.2 
Fig. 6.3 shows selecting $1^{\text {st }}$ application by pressing key 1 . In this result it is shown that for selecting $1^{\text {st }}$ application we have to press $1^{\text {st }}$ key from the keypad.

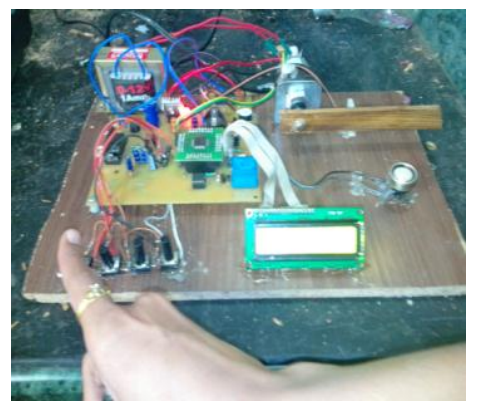

Fig.6.3

Fig.6.4 shows initialization of application. In this result, it is shown that we have to touch every time the iButton to iButton reader and then only we get correct output.

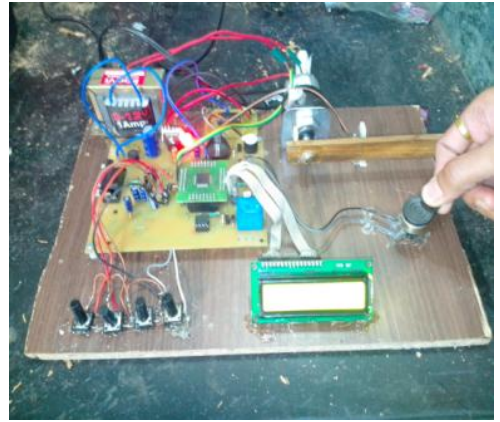

Fig.6.4

Result 6.5 shows identification. In this result it is shown that, after touching the iButton to reader, the account is matched properly and we get the correct detail.

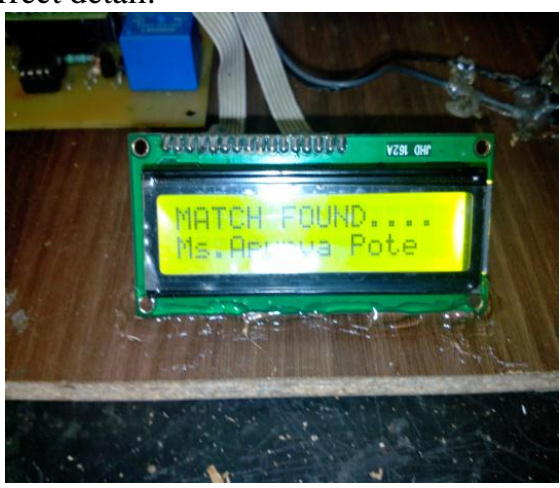

Fig.6.5

Fig. 6.6 shows authorization using aadhaar card. In this result it is shown that, after touching the iButton, the account is matched properly and we get the correct detail. On the basis of that detail we will get authorization.

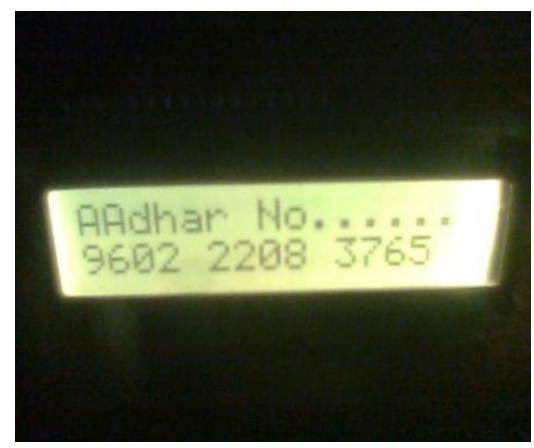

Fig.6.6 
Fig.6.7 shows gate is opened. This result shows that, after touching iButton to the iButton reader in the proper way, if the account is matched, immediately access will be given by the system.

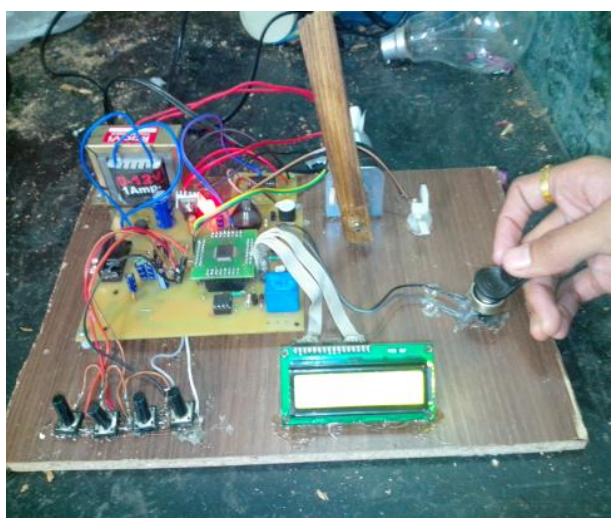

Fig. 6.7

Fig.6.8 shows that money is deducted from the account. This result shows that after touching the iButton to the reader, immediately access will be given by the system. And before giving access money is deducted from vehicle owner's account.

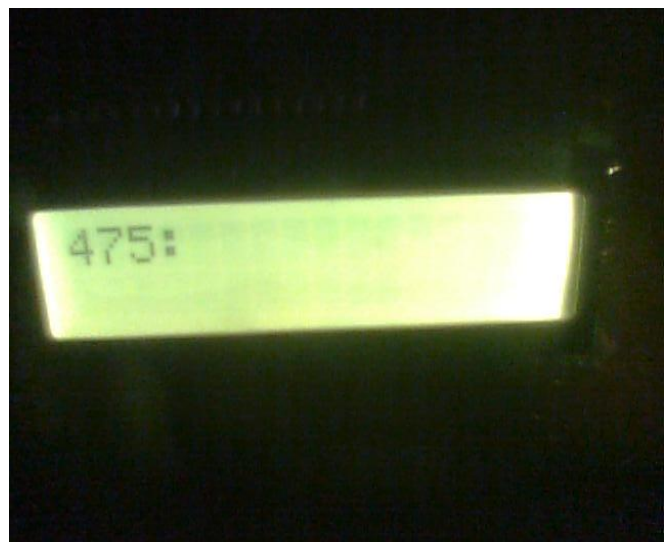

Fig.6.8

Fig.6.9 shows gate is closed after some time interval. In this result, it is shown that after giving access to the vehicle after some time interval gate will get closed automatically.

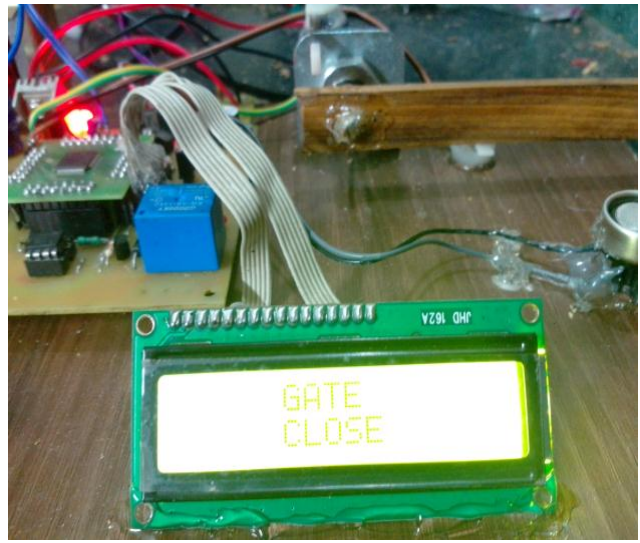

Fig.6.9 
Fig.6.10 shows registration details. In this result it is shown that after matching the account, if the account is matched then only it will display vehicle registration details and verification is performed.

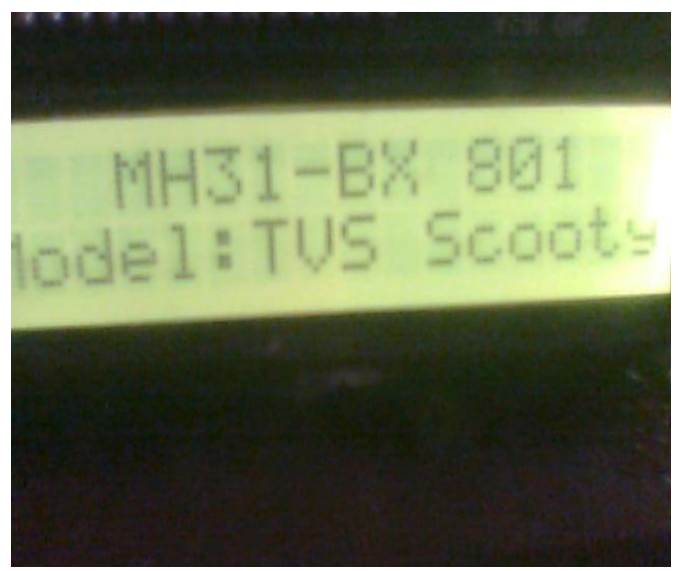

Fig.6.10

Fig 6.11 shows access is given to the vehicle.

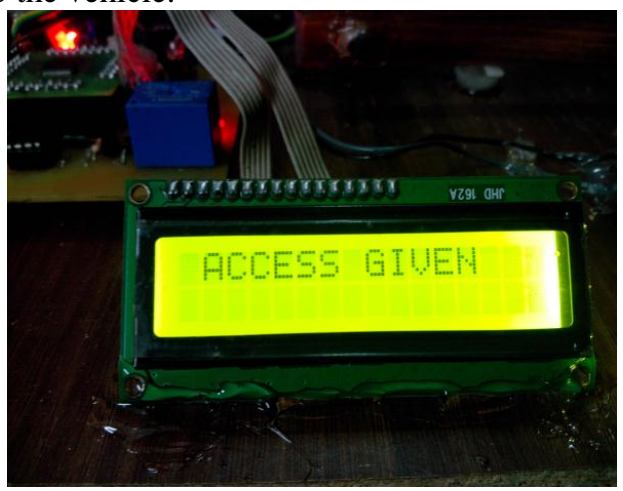

Fig.6.11

\section{Conclusion}

The hardware application of the 1-wire technology is described by this paper. The iButton devices are used in those cases where the information is transported by the person or an object. So, this can be used in the equipment, PC or building access. We can easily attach the device to mobile piece. We can discover many other diverse applications by searching on internet. The 1-wire network described in this paper is intended to be used for multitasking system which can serve three applications: Identification and authorization using aadhaar card, Toll access control, Vehicle registration details and access.

\section{References}

[1]. Eugen Diaconescu,Cristian Spirleanu,Identifying and authorizing application using ARM and 1- Wire technology, IEEE SIITME 978-1-4244-8124-8(2010).

[2]. Kai-Xin Tee,Moi-Tin Chew and Serge Demidenko,Intelligent Warehouse Stock Management and Tracking System Based on silicon identification technology, IEEE International Symposium on Electronic Design 978-0-7695-4306-2(2011).

[3]. Goran Martinovic,Tibor Kis-Konja,Zoran Kradija,Access control system based on electronic key, Technical Gazette 15(2008)2 3-7.

[4]. Miss.Bushra Anjum and Prof.S.Sharma, Access control and asset management using 1-wire technology for intelligent vehicle management system, ISBN: 978-93-81693-82-7.

[5]. Chengliang Zhang, Xianying Feng and Lei Li, The key technologies of a distributed temperature monitoring system based on 1-wire bus, Proc. $8^{\text {th }}$ IEEE World Congress on Intelligent Control and Automation, Jinan, China, July 6-9 2010.

[6]. B.Nkom, MIEEE, Concise schemes for realizing 1-wire cyclic redundancy checks, International Conference on adaptive science and technology, 11(8), 2011,4673-0759.

[7]. WAN Jian, YUAN Yi, WANG TaiYONG, Research and development of a portable data acquisition and analysis system based on arm and dsp, 10(6), 2010, 4244-5848.

[8]. Hongmei Xue, Research and development of an intelligent temperature-measuring system based on 1-wire bus, 8(5), 7695-3357, 2008.

[9]. Andrew Nowell, Remote computer interface \& security system (Secondary Research, iButton security system, Page-10)

[10]. MAXIM, Creating global identifiers using 1-wire devices (AN186, jan-2002).

[11]. MAXIM, Serial number iButton, (DS1990, 2008). 